\title{
Evaluation of Physical Activity Participation, Self-Efficacy and Outcome Expectancy for Employees Participating in Exercise Is Medicine ${ }^{\circledR}$ On Campus Program
}

\author{
Maximilian Gastelum-Morales*, Lisa J. Leininger ${ }^{*}$ Joanna L. Morrissey ${ }^{b}$, Ryan Luke ${ }^{a}$ \& Mark DeBeliso ${ }^{c}$ \\ ${ }^{a}$ Department of Kinesiology, California State University, Monterey Bay, Marina, CA \\ ${ }^{b}$ Department of Psychology, University of Wisconsin, Green Bay, Green Bay, WI \\ 'Department of Kinesiology and Outdoor Recreation, Southern Utah University, Cedar City, UT, United States \\ bttps:// doi.org/10.33697/ajur.2020.034
}

Student:Mgastelum-morales@csumb.edu

Mentor:Lleiningen@csumb.edu

\begin{abstract}
Exercise Is Medicine ${ }^{\circledR}$ On Campus (EIM-OC) is a worldwide initiative from the American College of Sports Medicine (ACSM) to promote physical activity (PA) at universities. California State University, Monterey Bay (CSUMB) implemented this initiative in Fall 2019 with offerings to students and employees. For employees, an "Introduction to Resistance Training Class" was offered. Participants attended classes two times per week, with the sessions lasting approximately fifty minutes. The purpose of this study was to evaluate the EIM-OC employee Introduction to Resistance Training class for its effectiveness on increasing PA, self-efficacy, and outcome expectancy. The research design was pre-post, with participants completing online questionnaires before and after the course. The Godin Leisure Time Physical Activity Questionnaire (LTPQ), Resistance Training Self-Efficacy and Outcome Expectancy Questionnaire, and Self-Efficacy and the Maintenance of Exercise Participation in Older Adults Questionnaire were included. The training class had a total of 14 female participants, 12 of which completed the pre- and postquestionnaires. There was a significant increase $(\mathrm{t}=-3.2, \mathrm{df}=11, \mathrm{p}=.004)$ in resistance training self-efficacy score following the course $(M=3.52 \pm 1.03$ versus $M=4.31 \pm .56)$. Resistance training outcome expectancy score was also statistically significant $(t=-$ $2.54, \mathrm{df}=11, \mathrm{p}=.01)$ following the course $(\mathrm{M}=4.48 \pm .53$ versus $\mathrm{M}=4.71 \pm .37)$. There were increases in strenuous exercise days, physical activity scores, and future resistance training self-efficacy, although they were not statistically significant. The results of this study indicate that employee exercise classes, as part of the EIM-OC initiative, can be effective in increasing resistance training self-efficacy, and outcome expectancy. These indicators are important for individuals to maintain lifelong PA therefore future programming and research on EIM-OC should continue.
\end{abstract}

\section{KEYWORDS}

Exercise Is MedicineR-On Campus; Resistance Training; Physical Activity; Exercise; Worksite Health Promotion Program; SelfEfficacy; Outcome Expectancy; Employees; California State University, Monterey Bay

\section{INTRODUCTION}

In the United States, individuals are dying prematurely due to lack of physical activity (PA) and an increase in non-communicable diseases. ${ }^{1}$ As PA levels decline, there is an increased risk of hypertension, obesity, diabetes, and many cancers. ${ }^{2}$ For example, in 2020, 1,806,590 new cancer cases and 606,520 cancer deaths are projected to occur in the United States. ${ }^{3}$ However, research indicates that patients involved in greater levels of exercise have a lower relative risk of cancer mortality and a lower relative risk of cancer recurrence, and they experience fewer and/or less severe treatment-related adverse effects. ${ }^{4}$ Furthermore, in healthy individuals, exercise can decrease the likelihood of succumbing to disease and illness. Considering physical inactivity across the globe, where only $26 \%$ of men and $19 \%$ of women report sufficient PA to meet the 150 -minute per week moderate aerobic activity and two day per week muscle-strengthening guidelines, it is important to implement strategies and programs to foster individual involvement in their own physical health. ${ }^{5}$

Studies have observed self-efficacy and its relationship with health behavior change. ${ }^{6}$ In social cognitive theory, ${ }^{7}$ self-efficacy is an important precondition for adequate self-management behavior. ${ }^{6}$ Increased self-efficacy levels have been shown to play a significant role in improving PA participatory patterns in adult females. ${ }^{8}$ Alongside self-efficacy, outcome expectancy and selfregulation have been identified to be highly associated with exercise behavior. A study done as part of a worksite intervention 
provided significant positive results for these three variables. ${ }^{9}$ Therefore, examining these variables in a university worksite population will add to the body of knowledge of the effects of programming on health behaviors.

Worksite health promotion programs (WHPPs) are an important tool due to its capacity to change the burden of physical inactivity, and it can serve to remove one challenge for those employees who deem PA to be out of their reach due to accessibility, instruction, or motivation. WHPPs have been shown to improve nutritional health behavior and increase PA in working class adults. ${ }^{10}$ Due to their numerous benefits, many universities have implemented WHPPs. ${ }^{11}$ In previous years California State University, Monterey Bay (CSUMB) implemented WHPPs such as the "Workplace Walk Off Challenge" and offerings from the insurance partners and local hospital wellness programs. ${ }^{12}$ WHPPs also play an integral part in exercise adherence for those employees who participate in them. ${ }^{9}$ Furthermore, WHPPs have been used by companies to keep employees healthy in order to prevent any health complications that would impede them from continuing to work. There is evidence that WHPPs are associated with lower levels of absenteeism and health care costs, and fitness programs are associated with reduced health care costs. ${ }^{13}$

\section{Exercise is Medicine $\mathbb{R}$ on Campus}

Exercise Is Medicine ${ }^{\circledR}$ is a global health initiative launched by the American Medical Association and the ACSM in 2007. The initiative aimed to make PA a standard of the medical paradigm for the prevention and treatment of non-communicable diseases in healthcare systems. Branching from this global health initiative is the Exercise Is Medicine ${ }^{\circledR}$ On Campus (EIM-OC) initiative, which encourages colleges and universities to promote PA to make movement part of the daily campus culture for both students and employees. EIM-OC activities help improve the knowledge and promotion of PA on college campuses. This is done in a variety of ways, and many departments are often involved (Kinesiology or related departments, Student Health Services, Student Counseling Services, and Health Promotion and/or Wellness Departments).

Many different programs were successfully implemented and have provided effective strategies for improving PA across campuses worldwide under the EIM-OC initiative. Effective strategies utilized by different campuses for a successful implementation of an EIM-OC program included placing exercise stations across campus to promote PA during an EIM-OC week, ${ }^{14,15}$ and using campus health centers to screen for inactivity in patients with the intention to refer them to an exercise practitioner that can help them with increasing their PA levels. ${ }^{16}$ Another major characteristic of a successful EIM-OC program is the amount of student involvement, students can play different roles such as promotion, leadership, organization, planning, education, implementation, and data collection. ${ }^{14-16}$ EIM-OC provides a strong framework due to the support and resources provided by the ACSM. EIM-OC allows campuses great flexibility to implement programming within their bandwidth and capabilities. However, fewer programs have included employees in their effort to increase PA on campus. Including employees is imperative if a campus wishes to truly have a culture of PA.

\section{Exercise is Medicine ${ }^{\circledR}$ on Campus at CSUMB}

CSUMB followed all steps necessary to register the campus and implement the EIM-OC program. This included creating a leadership team and completing the registration form. Concurrently, the leadership team developed campus support, and then implemented EIM-OC in a variety of ways, once registration was approved from the ACSM. During Fall 2019, the CSUMB EIMOC Executive Committee planned initial programming for students and employees. Employee programming focused on inperson exercise classes, which included an "Introduction to Resistance Training (RT) class." The RT class focused on employees learning to lift with proper technique, were given instant feedback on their performance, and were encouraged to work at their own pace so that they develop healthy behaviors towards resistance training inside and outside of the class. Given that RT can help maintain health in all populations, a goal for the program was to collect data on key health behavior variables in order to evaluate the effectiveness of the class.

PA level, self-efficacy and outcome expectancy were variables measured in the study due to their strong correlation to exercise health behaviors. It has been observed that high levels of PA have a significant effect on life expectancy. Studies have also found that self-efficacy and outcome expectancy are key psychological constructs which play a key role in exercise adherence, maintenance, and outcomes regarding strength training specifically. ${ }^{17}$ Self-efficacy is an individual's belief in oneself to execute situation-specific behavior and enhances an individual's behavioral persistence. ${ }^{18-20}$ Outcome expectancy is thought to play an important role in motivation and is defined as a person's belief that a specific behavior will lead to certain positive and negative consequences related to one's physical health or affective outcomes, such as impacts on one's mood or mental state. ${ }^{17,18,20}$

The research questions for this study were: 1) are there increases in PA participation among employees participating in the exercise classes offered through the CSUMB EIM-OC initiative? and 2) are there increases in exercise self-efficacy and outcome expectancy among employees who participate in the exercise classes offered through the CSUMB EIM-OC initiative? The 
purpose of this study was to evaluate the effectiveness of the EIM-OC employee Introduction to RT class, specifically related to PA participation, self-efficacy and outcome expectancy.

\section{METHODS AND PROCEDURES}

\section{Program Description}

Beginning in Fall 2019, EIM-OC the Introduction to RT class was made available to all campus employees (staff, faculty, administration). Space in the Kinesiology Department's exercise facility was limited; therefore, employees were required to sign up online through a link displayed on CSUMB's dashboard page. The Introduction to RT class was held on Tuesdays and Thursdays during lunch time hours (12pm to 12:50pm) for four weeks. Participants were introduced to a variety of RT exercises during the span of the course. Examples of exercises introduced included front squats, goblet squats, lunges, Romanian deadlifts, deadlift, bent over row, push-ups, military press, and dumbbell shoulder press. Participants primarily used free weights and learned to use proper exercise technique. Body weight exercises were also used to develop proper exercise technique in participants who showed the need. Equipment used included barbells, dumbbells, benches, rubber plates and clamps.

The class was led by a tenured Kinesiology professor who is a National Strength and Conditioning Association, Certified Strength and Conditioning Specialist. Undergraduate student leaders $(n=6)$, majoring in Kinesiology, were trained beforehand to assist in the facilitation of the classes. Student leaders set up, demonstrated exercises, and coached form. Employees who participated in this study were given clear instructions and information on what to expect, bring, and wear for the class. The Institutional Review Board at CSUMB approved this research study and all authors and researchers completed the Collaborative Institutional Training Initiative where they became certified to work with human subjects in research. Informed consent was obtained for all those participating in the research study. All participants filled out a Physical Activity Readiness Questionnaire (PAR-Q) to assure no contraindications for exercise participation.

\section{Participants}

No exclusion or inclusion criteria were established and the course was available to all employees (faculty, staff and administration) at the university. A participant cap of 16 was set due to space limitations. Fourteen employees volunteered for the study, with 12 completing both pre and post surveys, and therefore were included in the statistical analysis. No incentives were given for attendance or completion of surveys. Average age of the participants was 40.7 years $(\mathrm{SD} \pm 12.66)$. Of the 12 that completed both surveys, 10 were staff and two were faculty. All participants were female. Attendance was not mandatory, and participants were not required to participate in PA outside of the exercise intervention, but were encouraged to stay active. Out of the eight exercise sessions, five attended eight sessions, five attended six sessions, and two attended five sessions. Therefore, $41.6 \%$ of all participants completed all workout sessions, with $83 \%$ completing 6 or more sessions.

\section{Protocol}

Upon participant arrival to the class, a brief greeting was completed during which the exercises for the day were explained and demonstrated. After participants assembled into groups of two to three individuals, they began at a chosen station. Stations were set up in a circular fashion in order to increase supervision. Eight stations were available and prepped with the equipment for the session. Employees stayed at their station and student leaders circulated between the groups of employees, giving employees the opportunity to interact with each student leader during the session. Students provided verbal feedback to employees. Therefore, employees had significant guidance and support from the student leaders. Student leaders were responsible for arriving on time before each session and setting up. All six students were to attend all 8 sessions with exceptions made when necessary. Workouts were designed by the student leaders, and approved by the faculty lead. Exercises were chosen based on the recommendation of all major muscle groups being targeted during a given workout.

The research design was pre-post. Questionnaires were administered a week before the first class and two days following the last class in the four-week series. The online questionnaire included basic demographic information, the PAR-Q form, Godin Leisure Time Physical Activity Questionnaire, ${ }^{21}$ Resistance Training Self-Efficacy and Outcome Expectancy Questionnaire, ${ }^{22}$ and SelfEfficacy and the Maintenance of Exercise Participation in Older Adults Questionnaire.23

The Godin LTPQ includes a table in which participants indicate how many times on average they participated in the following intensity of exercise for more than 15 minutes during their free time in one week. Exercises intensities were broken into three categories: strenuous, moderate, mild/light. Scores were determined by taking the number for each category and multiplying it by a fixed variable, after which the scores for the three categories were added and interpreted through the questionnaire key where participants could be placed in three categories (active, moderately active, and insufficiently active/sedentary).

The Resistance Training Self-Efficacy \& Outcome Expectancy Questionnaire includes nine questions which are divided into two sections (Self-Efficacy and Outcome Expectancy). Four questions are regarding Self-Efficacy and five questions are regarding 
outcome expectancy. Answers for the questions were based on a five-point scale (one $=$ strongly disagree and five $=$ strongly agree). Scores were calculated by adding the answers and dividing them by the corresponding amount of questions. High levels of self-efficacy yielded a larger number, while lower yields indicated lower self-efficacy. Internal consistency for self-efficacy was $\boldsymbol{\alpha}=$ .83 , with internal consistency for outcome expectancy $\boldsymbol{\alpha}=.75 .^{22}$

The Self-Efficacy \& Maintenance of Exercise Participation in Older Adults Questionnaire included a set of eight questions which were scored based on a percentage scale which started at zero and ended at one-hundred $(0 \%=$ not at all confident, $50 \%=$ moderately confident, and $100 \%=$ Highly confident). Scores were derived by adding the scores and dividing by eight, high levels of self-efficacy yielded a larger number, while lower yields indicated lower self-efficacy. Internal consistency for this questionnaire was $\boldsymbol{\alpha}=.85$. While this questionnaire was originally designed for older populations, it has since been validated with other populations, including adults under the age of $65 . .^{24}$

\section{Statistical Analysis}

Paired-samples t-tests were performed for LTPQ score, strenuous days of PA, moderate days of PA, and light days of PA per week. Paired samples t-tests were also performed for resistance training self-efficacy, resistance training outcome expectancy, and resistance training future self-efficacy. All statistical analysis was done on SPSS version 25. Significance was set at $\boldsymbol{\alpha}=0.05$.

\section{RESULTS}

Resistance training self-efficacy score was statistically significant $(\mathrm{t}=-3.2, \mathrm{df}=11, \mathrm{p}=.004)$ from pre $(\mathrm{M}=3.5 \pm 1.03)$ to post $(\mathrm{M}=4.31 \pm .56)$ RT class. Resistance training outcome expectancy score was also statistically significant $(\mathrm{t}=-2.54, \mathrm{df}=11, \mathrm{p}=.01) \mathrm{pre}$ $(\mathrm{M}=4.48 \pm .53)$ to post $(\mathrm{M}=4.71 \pm .37) \mathrm{RT}$ class. See Figures $1 \& 2$.

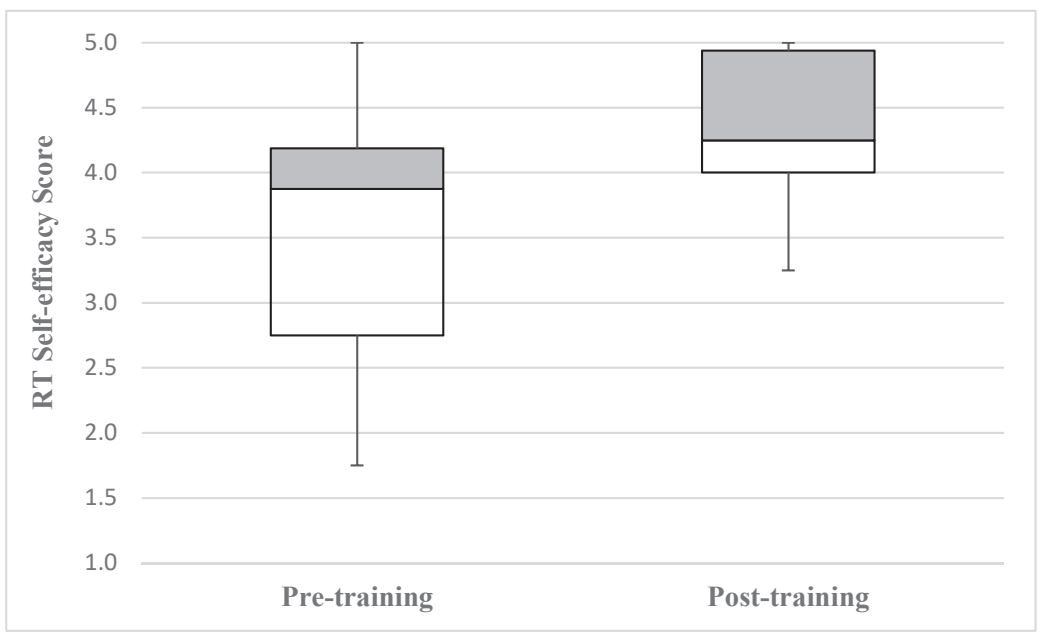

Figure 1. Resistance training self-efficacy score pre and post training. $\mathrm{p}<0.05$

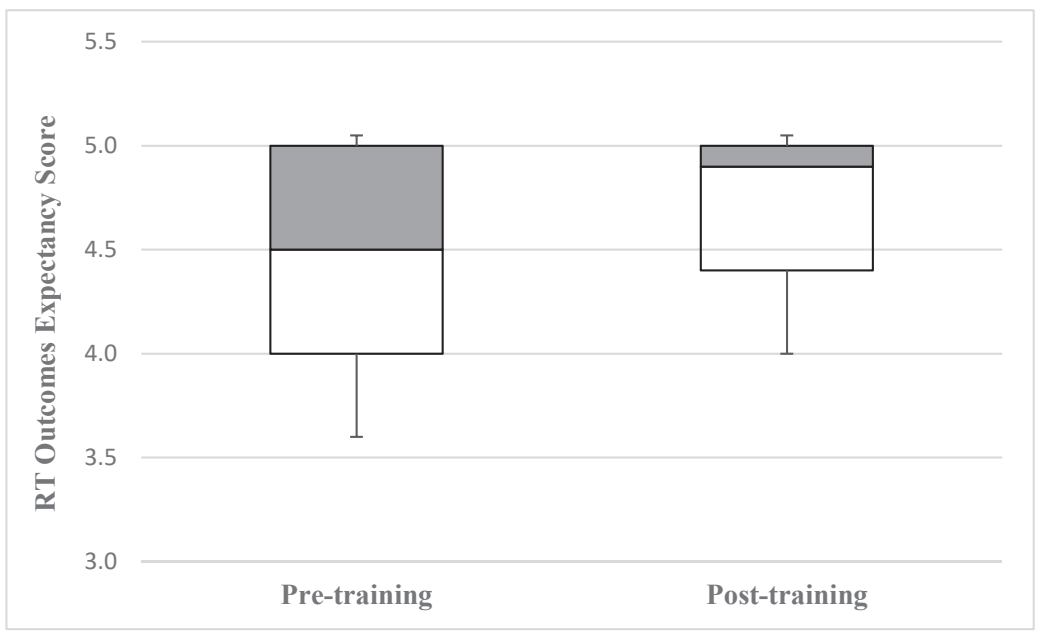

Figure 2. Resistance training outcome expectancy score pre and post training. $\mathrm{p}<0.05$ 
Future resistance training self-efficacy was not statistically significant $(\mathrm{t}=-.06, \mathrm{df}=11, \mathrm{p}=.48)$, although there was an increase from pre $(\mathrm{M}=89.06 \pm 12.58)$ to post $(\mathrm{M}=89.27 \pm 15.13)$ training. See Figure 3.

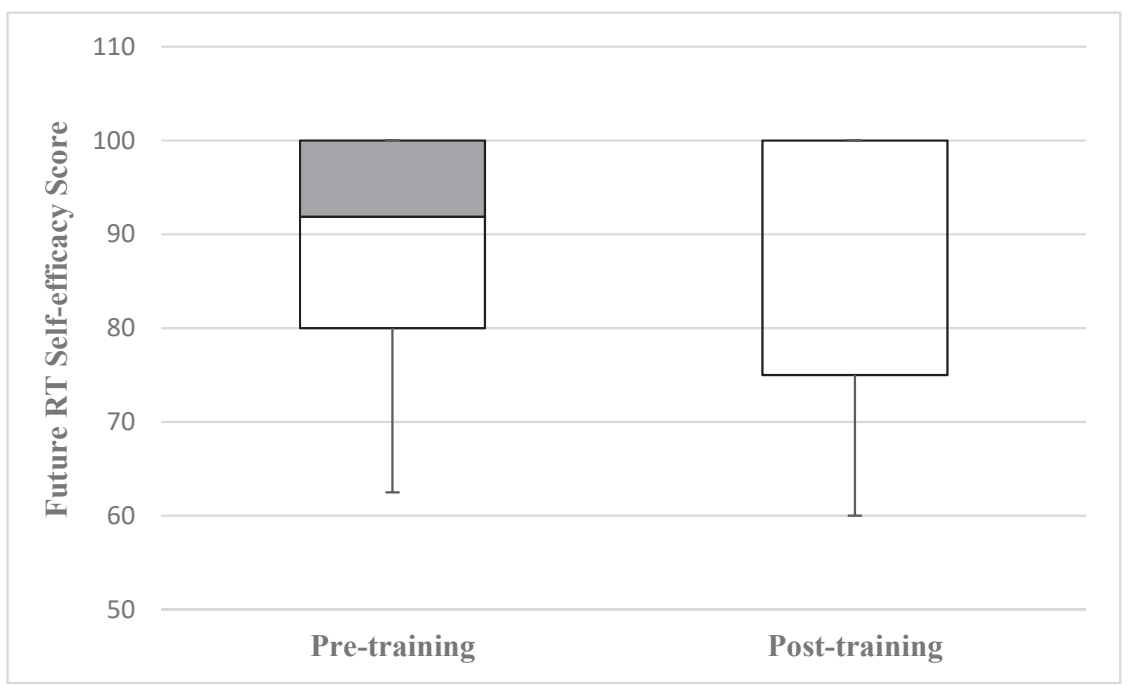

Figure 3. Future resistance training self-efficacy score.

LTPQ PA Score was not statistically significant, but also increased $(\mathrm{t}=-.54, \mathrm{df}=11, \mathrm{p}=.30)$ from pre $(\mathrm{M}=44.08 \pm 27.9)$ to post $(\mathrm{M}=46.58 \pm 21.73)$. Days of strenuous PA per week were not statistically significant, but increased $(\mathrm{t}=-\mathrm{9} .59, \mathrm{df}=11, \mathrm{p}=.18)$ from pre $(\mathrm{M}=1.67 \pm 1.87)$ to post $(\mathrm{M}=2.08 \pm 1.73)$. Days of moderate PA per week were not statistically significant and unchanged $(\mathrm{t}=.00, \mathrm{df}=11, \mathrm{p}=.50)$ from pre $(\mathrm{M}=3.42 \pm 2.15)$ to post $(\mathrm{M}=3.42 \pm 1.37)$. Days of light PA per week were not statistically significant $(\mathrm{t}=1.0, \mathrm{df}=11, \mathrm{p}=.16)$ from pre $(\mathrm{M}=4.0 \pm 2.0)$ to post $(\mathrm{M}=3.58 \pm 2.23)$.

See Figure 4.

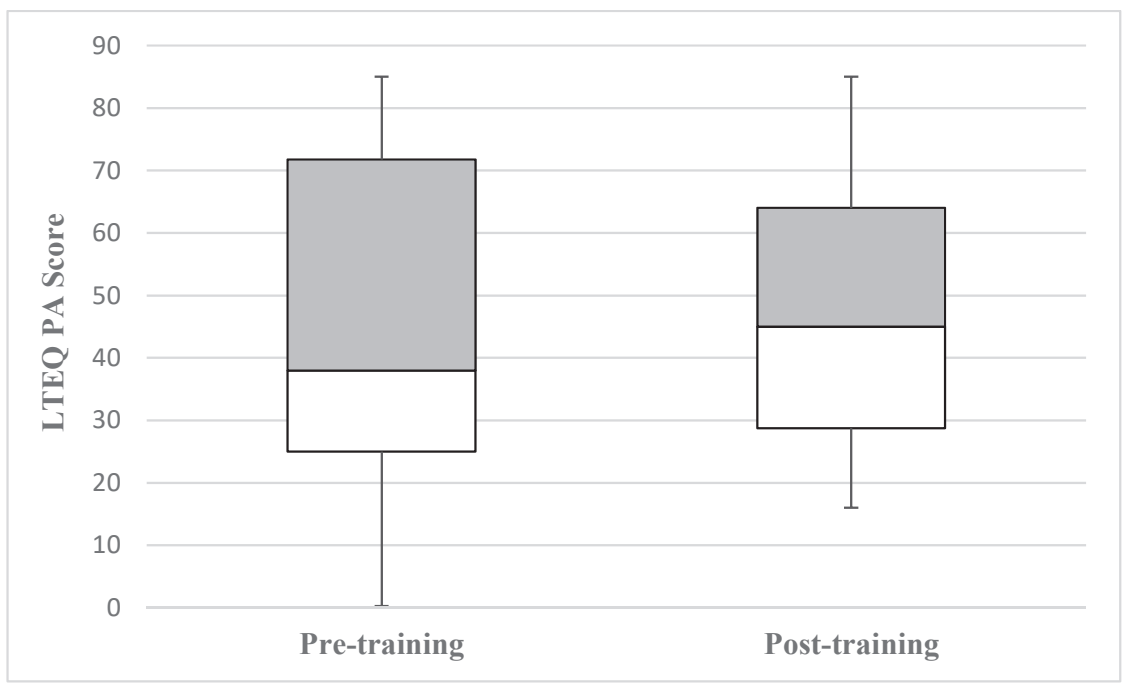

Figure 4. LTPQ score pre versus post training.

Given the appearance of the box plots, Wilcoxon signed-rank tests were conducted for each of the dependent variables. The results of the Wilcoxon analysis were consistent with the paired samples t-tests for each dependent variable. 


\section{DISCUSSION}

The purpose of this study was to evaluate the effectiveness of the EIM-OC employee Introduction to RT class. This course was implemented as part of the EIM-OC initiative at CSUMB. This study focused on the effects of an employee RT class on PA participation, resistance training self-efficacy and outcome expectancy.

Results of this study indicated that the program's intervention was effective in influencing health behavior variables. Significant changes in self-efficacy and outcome expectancy were observed. In addition, increasing trends in PA score, PA days (light, moderate, and strenuous) and future RT self-efficacy were observed. While university employees are a small sample of the population, the effectiveness of WHPPs can prove effective in maintaining their health to avoid any unwanted health emergencies or complications associated with the decline in PA. ${ }^{25}$

This study provided evidence to the effectiveness of the EIM-OC employee Introduction to RT class, WHPPs and the EIM-OC program at CSUMB in improving key psychological constructs that play a role in exercise adherence. Previous studies that have tracked self-efficacy and outcome expectancy have found that through an exercise intervention they can be positively influence. ${ }^{7,8}$ Studies have also shown that improved self-efficacy in adults correlates to gait speed which is an important predictor of current and future functional status. ${ }^{26}$ This study provides evidence that exercise interventions can impact self-efficacy, which in of itself has been a proven predictor of health quality. Furthermore, it is adding literature to the growing body of knowledge regarding EIM-OC. As of this study's publication, most EIM-OC programs focus on student programming. However, to truly make physical activity at part of the campus culture, employees should be included. The program at CSUMB differs from many other EIM-OC programs because it is invested in developing exercise interventions for employees. Therefore, this EIM-OC program provides a framework to implement a worksite health promotion program.

While this study required plenty of planning, background knowledge and training, it was feasible with a team of individuals who have proper experience in research and implementing WHPPs. Proper steps were taken to ensure that employees in the study were safe and properly informed of their options.

The employees participating in the study went through a four-week intervention in which they were provided with two days of guided exercise instruction. Each day incorporated exercises which stressed all major muscle groups. No incentives were given for attendance, but it was recommended that participating employees attend both days. A support system made up of student leaders and a certified professional served to guide, motivate, and encourage participating employees.

\section{Limitations}

There are some study limitations to note. A participant cap was set at 16, due to limited space within the exercise science lab. Due to class limitations, the sample size $(n=12)$ was small. Additionally, the participants were all female and mostly staff members. Therefore, these results may not be generalizable to the larger campus population or more diverse groups of employees who participate in future studies.

The questionnaires used required participants to self-report data which could have impacted the validity of the results. For example, given the pre- and post-structure of filling out questionnaires, participants might have an inaccurate recollection of their daily activity over the four weeks of the study. With attendance not mandatory, employees may not have attended all classes potentially impacting the results of the study.

The participants who volunteered for this study were also already considered active. Of the 14 individuals who began the study, 11 were rated as "Active" according to the LTPAQ, while three were considered "Insufficiently Active." Following the RT course, of the 12 participants who completed the study, 10 were scored as "Active" and two were scored as "Moderately Active." No participants were scored as "Insufficiently Active" following the course. Additionally, this study did not have a control group, which could have helped demonstrate that the three variables being measured could have been impacted by other factors.

\section{Implications and Areas of Future Research}

Future EIM-OC employee Introduction to RT classes should aim to increase participation among employees, especially related to recruiting males. Issues facing EIM-OC during this pilot study were having a small sample size, lack of accountability by the participants, coupled with a short intervention period of four weeks. This study also only had one population which was involved, adult females. Participants in this study participated out of their own interest. Participants were encouraged to attend both classes each week, but there were no repercussions if they did not make one of the sessions. The choice to participate in the study was also based on personal preference. There were participants who filled out both pre and post surveys, which ultimately made it to the results, however there were also individuals who filled out one survey or did not fill out any surveys while still participating in the exercise intervention. 
Given that this study was a pilot and the exercise science lab which was used had limited space, a small sample size was effective in allowing the management and implementation of the RT class. Future research should look into finding larger spaces which would allow for more participants. In addition, the program should try to advertise the classes better in order to diversify the population which is involved in the study. Tactics that can be used to increase diversity and participation include incentives, promotional videos on campus, availability of classes, and cross campus communication.

Future research should address these issues in order to maximize the effectiveness of the employee Introduction to RT Class. Increasing the number of participants and diversifying the population of the study would allow for generalizability within different populations. Increasing the length of the study would allow for a more in-depth view into the key psychological constructs which play a role in exercise adherence (outcome expectancy and self-efficacy). These factors should be considered when planning the employee Introduction to RT Class for upcoming terms.

\section{CONCLUSION}

The purpose of this study was to evaluate the effectiveness of the EIM-OC employee Introduction to RT class. The results demonstrate that the program was effective in increasing key psychological constructs which related to exercise adherence and participation. A four-week 2 session per week intervention of RT showed positive increases in self-efficacy and outcome expectancy. Further studies should be conducted in order to provide more evidence for the effectiveness of the EIM-OC employee RT class. This study adds to the growing body of knowledge that currently exists on EIM-OC and WHPPs. Through effort on part of the EIM-OC team at CSUMB the program was successful, although different results might be attained in a more diverse population.

\section{ACKNOWLEDGEMENTS}

The authors thank the Undergraduate Research Opportunity Center (UROC) and the McNair Scholars Program for helping fund this project. This project would not be feasible without the Kinesiology department and Associate Professors supporting the process of planning and implementation of the program, along with mentorship of student leaders.

\section{REFERENCES}

1. Danaei, G., Ding, E. L., Mozaffarian, D., Taylor, B., Rehm, J., Murray, C. J., \& Ezzati, M. (2009) The preventable causes of death in the United States: Comparative risk assessment of dietary, lifestyle, and metabolic risk factors. PLoS Medicine, 6(4), 123. doi:10.1371/journal.pmed.1000058

2. Cunningham, C., Sullivan, R. O., Caserotti, P., \& Tully, M. A. (2020) Consequences of physical inactivity in older adults: A systematic review of reviews and meta-analyses. Scandinavian Journal of Medicine \& Science in Sports, 30(5), 1-12. doi: $10.1111 /$ sms.13616

3. Siegel, R. L., Miller, K. D., \& Jemal, A. (2020) Cancer statistics, 2020. CA: A Cancer Journal for Clinicians, 70(1), 7-30. doi: 10.3322 / caac. 21590

4. Cormie, P., Zopf, E. M., Zhang, X., \& Schmitz, K. H. (2017) The impact of exercise on cancer mortality, recurrence, and treatment-related adverse effects. Epidemiologic Reviews, 39(1), 71-92. doi: 10.1093/ epirev/ mxx007

5. U.S. Department of Health and Human Services. Physical Activity Guidelines for Americans, 2nd edition, https:// health.gov/sites/default/files/2019-09/Physical_Activity_Guidelines_2nd_edition.pdf(accessed June 2020)

6. Sol, B. G., Graaf, Y. V., Petersen, R. V., \& Visseren, F. L. (2011) The effect of self-efficacy on cardiovascular lifestyle. European Journal of Cardiovascular Nursing, 10(3), 180-186. doi:10.1016/j.ejcnurse.2010.06.005

7. Bandura, A. (1998) Health promotion from the perspective of social cognitive theory. Psychology \& Health, 13, 623-649. doi:10.1080/08870449808407422

8. Mcauley, E., \& Jacobson, L. (1991) Self-efficacy and exercise participation in sedentary adult females. American Journal of Health Promotion, 5(3), 185-207. doi:10.4278/0890-1171-5.3.185

9. Hallam, J. S., \& Petosa, R. (2004) The long-term impact of a four-session work-site intervention on selected social cognitive theory variables linked to adult exercise adherence. Health Education \& Behavior, 31(1), 88-100. doi:10.1177/1090198103259164

10. Mache, S., Jensen, S., Jahn, R., Steudtner, M., Ochsmann, E., \& Preuß, G. (2015) Worksite health program promoting changes in eating behavior and health attitudes. Health Promotion Practice, 16(6), 826-836. doi:10.1177/1524839915596310

11. Hill-Mey, P., Hyatt-Neville, B., Kumpfer, K., Merrill, R., Reel, J., \& Richardson, G. (2015) Worksite health promotion programs in college settings. Journal of Education and Health Promotion, 4(1), 1-7. doi:10.4103/2277-9531.154019

12. Leininger, L. J., Dames, K. D., Adams, K. J., Climstein, M., \& Beliso, M. D. (2019) Positive impacts of a university walking program: A case study. Journal of Physical Activity Research, 4(1), 57-61. doi:10.12691/jpar-4-1-7

13. Aldana, S. G. (2001) Financial impact of health promotion programs: A comprehensive review of the literature. American Journal of Health Promotion, 15(5), 296-320. doi:10.4278/0890-1171-15.5.296 
14. Bopp, M., Bopp, C. M., Duffey, M. L., Ganim, R., \& Proctor, D. N. (2015) Implementation and evaluation of an Exercise is Medicine ${ }^{\mathrm{TM}}$ on campus week. Evaluation and Program Planning, 52, 176-181. doi:10.1016/j.evalprogplan.2015.06.003

15. Melton, B., Williamson, J. A., Bland, H., \&amp; Zhang, J. (2016) Using the Exercise is Medicine $\mathbb{R}$ on Campus platform to assess college students' practice of physical activity in a rural setting. Journal of the Georgia Public Health Association, 5(4), $360-364$. doi:10.21633/jgpha.5.402

16. Lynn, J, Urda, J, Winters, C (2015)Slippery Rock University A Case Study of Success in Exercise is Medicine ${ }^{\circledR}$ On Campus, ACSM's Health \& Fitness Journal, 19(4), 34-36. doi: 10.1249/FIT.0000000000000130

17. Hadley, K., \& Morrissey, J. (2019) Psychological constructs as predictors of strength gains in a strength training course. American Journal of Undergraduate Research, 16(1), 41-48. doi: 10.33697/ ajur.2019.015

18. Rodgers, W. M., Markland, D., Selzler, A., Murray, T. C., \& Wilson, P. M. (2014) Distinguishing perceived competence and self-efficacy: An example from exercise. Research Quarterly for Exercise and Sport, 85, 527-539. doi:10.1080/02701367.2014.961050

19. Bandura, A. (1977) Self-efficacy: Toward a unifying theory of behavioral change. Psychological Review, 84(2), 191-215. doi:10.1037/0033-295x.84.2.191

20. Williams, D. M., Anderson, E. S., \& Winett, R. A. (2005) A review of the outcome expectancy construct in physical activity research. Annals of Behavioral Medicine, 29(1), 70-79. doi:10.1207/s15324796abm2901_10

21. Godin, G. (2011) The Godin-Shepard Leisure-Time Physical Activity Questionnaire. Health and Fitness Journal of Canada, 4 (1), $18-22$.

22. Lubans, D. R., Aguiar, E. J., \& Callister, R. (2010) The effects of free weights and elastic tubing resistance training on physical self-perception in adolescents. Psychology of Sport and Exercise, 11(6), 497-504.

23. McAuley, E. (1993) Self-efficacy and the maintenance of exercise participation in the older adults. Journal of Behavioral Medicine, 16, 103-113.

24. McAuley, E., Lox, C., Duncan, T. E. (1993) Long-term maintenance of exercise, self-efficacy and psychological change in older adults. Journal of Gerontology: Psychological Sciences, 48(4), 218-224.

25. Knight, J. A. (2012) Review of physical inactivity: Associated diseases and disorders. Annals of Clinical \& Laboratory Science, 42(3), 320-337.

26. Liu-Ambrose, T., Davis, J. C., Nagamatsu, L. S., Hsu, C. L., Katarynych, L. A., \& Khan, K. M. (2010) Changes in executive functions and self-efficacy are independently associated with improved usual gait speed in older women. BMC Geriatrics, 10(1) doi:10.1186/1471-2318-10-25

\section{ABOUT STUDENT AUTHOR}

Maximilian Gastelum-Morales will graduate from California State University, Monterey Bay in Fall 2020 with a Bachelor's degree in Kinesiology - Exercise Science. Maximilian is a McNair Scholar and an EIM-OC student leader. Max has had the opportunity to present research findings at the Hawaii International Conference on Education in January 2020, and the ACSM annual conference in May 2020. His future career goals include being a researcher professor within the field of Kinesiology. Areas of study that interest him include biomechanics, physiology, strength \& conditioning, and sports medicine.

\section{PRESS SUMMARY}

Exercise is Medicine on Campus (EIM-OC) is a worldwide health initiative within higher education institutions. CSUMB's EIMOC program included an Introduction to Resistance Training class which was offered to all employees. The research design was pre/post; Surveys were completed before the start of the first class and after the end of the last class. Results of this study indicate that employees who participated in EIM-OC improved upon Resistance Training Self-Efficacy and Outcome Expectancy, two variables which are related to exercise adherence. This study adds to the evidence that worksite exercise programs, including RT, can be effective at improving exercise adherence within female employees. 